Portland State University

PDXScholar

$11-15-2017$

\title{
Body Changes with Baby: a Qualitative Insight into Body Image After Birth
}

Samantha Lewis

Portland State University

Follow this and additional works at: https://pdxscholar.library.pdx.edu/honorstheses Let us know how access to this document benefits you.

\section{Recommended Citation}

Lewis, Samantha, "Body Changes with Baby: a Qualitative Insight into Body Image After Birth" (2017). University Honors Theses. Paper 486.

https://doi.org/10.15760/honors.490

This Thesis is brought to you for free and open access. It has been accepted for inclusion in University Honors Theses by an authorized administrator of PDXScholar. Please contact us if we can make this document more accessible: pdxscholar@pdx.edu. 
Body Changes with Baby: A Qualitative Insight into Body Image After Birth

$$
\text { by }
$$

Samantha Lewis

An undergraduate honors thesis submitted in partial fulfillment of the requirements for the degree of

\author{
Bachelor of Arts \\ in \\ University Honors \\ and \\ Psychology \\ Thesis Adviser \\ Christopher Allen, Ph.D.
}

Portland State University 


\begin{abstract}
Pregnancy is stressful to a woman's body and often leaves physical reminders such as stretch marks, loose skin, or scars that last for many months or years after giving birth. This study used semi-structured interviews and an inductive qualitative approach to interview eight participants and explore some of the methods women uses to cope with body changes from pregnancy. Analysis of interview transcripts revealed the most common changes experienced during pregnancy were weight gain, hair growth, and stretch marks; after pregnancy, weight loss, hair loss, and sagging breasts were the most common changes. The dominant coping strategy cited by participants was normalization; reaffirmation from the baby or child and purchasing better fitting clothes were additional coping strategies. Clinical implications of these findings include the need for better support systems for mothers who experience distress due to body changes during pregnancy and after birth.
\end{abstract}




\section{Introduction}

Pregnancy can have lasting impacts on a mother. The changes that accompany pregnancy are multi-dimensional, and include attitudinal, perceptual, and cognitive shifts, as well as changes in a complex construct called 'body image' (see Cash \& Pruzinsky, 1990; Thompson, Heinberg, Altage, \& Tantleff-Dunn, 1999; Tiggemann, 2004). Body image is the internal representation of one's outer appearance and is influenced by many factors, especially changes in the body's physical appearance. During pregnancy, a woman's body adapts to the fetus growing within her and changes as necessary. Visual changes may include weight gain, stretch marks, rounded and protruding abdomen, fuller hair, glowing skin, and enlarged breasts. Less public changes may include indigestion, morning sickness, headaches, changes in appetite, difficulty sleeping, pelvic pain, gestational diabetes, and preeclampsia. In addition, there may be an overall decrease in mobility and physical abilities, as well as an impact on quality of life (Hueston \& Kasik-Miller, 1988).

Beyond the bodily changes experienced during gestation, many women also express concern for their ability to "bounce back" quickly after birth and erase the evidence of their childbearing body (Nash, 2012). Women often feel that their postbirth body is drastically different from their prebirth body, and it can take a long time for the body to return to its prebirth weight and shape. For many women though, despite healthy eating and exercise habits, this never happens, and can lead to distress and frustration. While some women report positive body attitudes after giving birth, other studies have shown that there is an increase in body dissatisfaction after giving birth (Clark, Skouteris, Wertheim, Paxton, \& Milgrom, 2009a; Rallis, Skouteris, Wertheim, \& Paxton, 2007). 
Not only must a woman face physical changes with pregnancy, but also cognitive, emotional, relationship, and self-identification changes. Often times when a woman enters the postbirth period, her body is far from the thin-ideal that has become normalized in society, which can lead to body dissatisfaction (Gow, Lydecker, Lamanna, \& Mazzeo, 2012). Many new mothers may feel alone during the postpartum period, leading to struggles with feelings of failure, doubt, and shame as a result of being unable to identify with the "motherhood mystique:" a societally adopted mystique that asserts being a mom is easy and enjoyable (Hoffnung, 1989).

Magazines and television frequently glorify pregnancy by upholding women who maintain both their physique and sex appeal, creating a tremendous amount of pressure on women to maintain their figures. Mass media often celebrates women who push their physical limits while carrying a child, referencing those who continue to run marathons as "heroines" or "superheroes" (Nash, 2011). These media influences can lead to changes in self-perception and body image at any point of pregnancy, and after birth (Gow, Lydecker, Lamanna, \& Mazzeo, 2012).

After a baby is born, the mother's body typically has visual evidence of a previous pregnancy. For some women, the evidence is visible and clear (e.g. loose skin, extra weight, hair loss, scar from Caesarean delivery, etc...); however, for many mothers, there can be internal and invisible changes as well (e.g. postpartum depression, fibroids, hernia, vaginal tearing or lacerations from birth, rectocele, etc...). The internal changes often require many months, if not years, to heal, and some have lifelong consequences (Patel, Lee, Wheatcroft, Barnes, \& Stein, 2005). Research has shown that weight retention often has the greatest psychological impact on mothers (Jenkins \& Tiggemann, 1997; Walker, 1998). 
Previous studies aim to collect information on body dissatisfaction (e.g. Clark, Skouteris, Wertheim, Paxton, \& Milgrom, 2009b; DePietro, Millet, Costigan, Gurewitsch, \& Caulfield, 2003) however this study focused more on overall experience of body change during and after pregnancy. While the research on postbirth bodily acceptance has become increasingly diverse in methods of data collection and analysis, there is only a small amount of research on body changes beyond the postpartum timeframe (see Jordan, Capdevila, \& Johnson, 2005). The existing research has limited the postpartum period to a short period of time; generally, the postpartum period is defined as anywhere from six weeks to twelve months after giving birth, although one qualitative study did lengthen the postpartum period to three-years (Clark, Skouteris, Wertheim, Paxton, \& Milgrom, 2009b; Jordan, Capdevila, \& Johnson, 2005; Rallis, Skouteris, Wertheim, \& Paxton, 2007).

The aim of this study was to explore some of the methods women use to cope with body changes endured during and after pregnancy, in a post-birth time period greater than previous studies with the use of qualitative methodology. The decision to extend the postpartum frame to ten years was made under the preliminary assumption that bodily acceptance is not always a process limited to a few weeks or months. However, to ensure that the memories of pregnancy and the postpartum period are still accurate, the timeframe has been limited to ten years postbirth. While there are many studies that have explored the postpartum period, only a handful of studies have examined body changes using qualitative methodology (see Clark, Skouteris, Wertheim, Paxton, \& Milgrom, 2009a, Jordan, Capdevila, \& Johnson, 2005, Patel, Lee, Wheatcroft, Barnes, \& Stein, 2005). The use of qualitative methodology in this study allowed for flexibility in analysis, and often reveals different information than is gathered with quantitative methods (Grbich, 2007). 
For the sake of this study, the prebirth period is defined as the time in a women's life prior to giving birth, including gestation. The postbirth period is the remainder of a women's life after she has given birth for the first time. The number of births a woman has undergone in her lifetime was not taken into account in this study. Prior research has focuses on postpartum depression and dissatisfaction with the maternal body, while this study examines overall experience with pregnancy and postbirth (see Clark, Skouteris, Wertheim, Paxton, \& Milgrom, 2009b, Devine, Bove, \& Olson, 2000; Jordan, Capdevila, \& Johnson, 2005; Rallis, Skouteris, Wertheim, \& Paxton, 2007).

\section{Method}

\section{Participants}

A total of 8 participants were recruited via word of mouth by the research team. All participants were women who had given birth to at least one child within the last ten years. The sample of participants was a convenience sample; the research team invited those they knew were a good fit for the study to participate. In order to protect participant identity and decrease the risk of a confidentially breach, demographic information was not collected; however, most participants mentioned that they were married at the time of conception and birth, and resided on the west coast of the United States.

\section{Design and Procedure}

All aspects of this research were approved by the Institutional Review Board at Portland State University (protocol \#174299). Taking an inductive qualitative approach, this study used in-depth semi-structured interviews to gain insight into postbirth body changes experienced by 
women. Participants were recruited by the research team using the recruitment transcript (see Appendix A) and provided with a copy of the consent form (see Appendix B) at the time of interview scheduling and again prior to the start of the interview.

Participants were offered the options of either attending an in-person interview in a private office in Portland, OR, or to take part in a remote interview via Skype or FaceTime. All participants elected to have remote interviews, citing convenience as the primary reason for this decision. These remote interviews were conducted from the researcher's private home office to better ensure privacy and confidentiality. The researcher used casual language to allow for a natural flow of conversation with the participant, and all interviews lasted approximately 20 to 30 minutes. The interview used three main prompts and questions (see Appendix C) as well as improvised follow up questions that suited the conversation with and information from the participant. The interview guide also served as a note taking form for the researcher during the interview. No compensation was offered for participating in this research; however, participants had the option to include their email address on the consent such that they may receive a copy of the final study and results. At the end of the interview, participants were for thanked for their time and willingness to take part in this study. Interviews were recorded using standard Android recording software and were transcribed by the researcher within 4 days of the interview's occurrence.

\section{Data Analysis}

Transcripts were first coded for symptomology of pregnancy and postbirth. Transcripts were also analyzed using thematic content analysis, which provides different data than quantitative analytic coding. By developing a code for identifying themes in text data, thematic 
content analysis allows for a subjective interpretation of interview findings (Hsieh \& Shannon, 2006; Neuendorf, 2002). Interview transcripts were read multiple times by the researcher, with each read serving a different purpose in analysis. The first reading of the transcripts was used to develop a basic and simple understanding of the participant's bodily experiences during pregnancy and in the postbirth period. Subsequent and repeated transcript readings allowed for the emergence of different themes and specific concepts, leading to the establishment of further categories. The final readings were looking for specific examples and interview quotations that illustrated similar experiences amongst the participants.

\section{Results}

\begin{tabular}{|r|c|}
\hline \multicolumn{2}{|c|}{ Changes During Pregnancy } \\
\hline Change & Occurence \\
\hline Weight Gain & 8 \\
\hline Hair Growth & 7 \\
\hline Stretch Marks & 6 \\
\hline Other Physical Symptoms & 6 \\
\hline Water Retention/Swelling & 5 \\
\hline Hair Texture & 4 \\
\hline Englarged Breasts & 4 \\
\hline Cramping & 3 \\
\hline Pregnancy Complications & 3 \\
\hline Hemorrhoids & 2 \\
\hline Hernia & 1 \\
\hline
\end{tabular}

Table 1: Changes experienced by participants during pregnancy.

\begin{tabular}{|r|c|}
\hline \multicolumn{2}{|c|}{ Changes After Pregnancy } \\
\hline Change & Occurence \\
\hline Hair Loss & 7 \\
\hline Weight Loss & 7 \\
\hline Decrease in Breast Fullness & 6 \\
\hline Difficulty Loosing Weight & 4 \\
\hline Mom "Pooch" & 4 \\
\hline Complications from Birth & 4 \\
\hline Hair Texture & 3 \\
\hline Breasts Remain Enlarged & 3 \\
\hline Anxiety/Depression & 3 \\
\hline Increase in Stress & 2 \\
\hline Skin Changes & 2 \\
\hline No Time for Self & 2 \\
\hline Overall Body Shape Change & 1 \\
\hline
\end{tabular}

Table 2: Changes experienced by participants after pregnancy.

Initial coding of symptomology revealed that during pregnancy, all participants experienced significant weight gain (significantly was defined as a gain greater than 20lbs), and most all experienced additional hair growth $(\mathrm{N}=7)$ and formed stretch marks $(\mathrm{N}=6)$ (see full symptom details in Table 1). After giving birth, most women subsequently experienced hair loss 
$(\mathrm{N}=7)$ as well as weight loss $(\mathrm{N}=7)$, and most participants $(\mathrm{N}=6)$ mentioned that sagging or "deflated" breasts were one of the most prominent body changes they experienced, citing breastfeeding as the primary reason for this change (see full symptom details in Table 2). All participants who experienced a change in breast size and/or fullness in the postbirth period associated the decrease with the ending of breastfeeding, and described the change in breast shape to be rapid disappointing.

When asked about coping strategies that helped them deal with the aforementioned body changes, most women described normalization of their bodies $(\mathrm{N}=7)$ as the most useful coping strategy. Many participants also cited support from their family and friends (which include their partner in every case) $(\mathrm{N}=6)$, as well as purchasing better

\begin{tabular}{|c|c|}
\hline \multicolumn{2}{|l|}{ Coping Strategies } \\
\hline Strategy & Occurence \\
\hline Normalization & 7 \\
\hline Family/Friend Support & 6 \\
\hline Purchasing Fitting Clothing & 5 \\
\hline Reaffirmaiton of 'Mother' Role & 4 \\
\hline Talking to Other Moms & 4 \\
\hline Lifestyle Changes & 3 \\
\hline Research & 3 \\
\hline Medication & 2 \\
\hline Counseling & 1 \\
\hline Knowing Somebody Has It Worse & 1 \\
\hline Home Remedies & 1 \\
\hline Photographing Pregnancy & 1 \\
\hline
\end{tabular}

Table 3: Coping strategies used by participants. fitting clothes for their postbirth body $(\mathrm{N}=5)$ as helpful coping techniques. Participants mentioned a total of 12 different coping methods.

As noted by some participants, the use of the Internet and social media may have an influence on the experience of body changes after giving birth. As specifically mentioned by participant 5:

"I think having peer groups you know, Facebook, is huge.... there was a mom's group that all had kids born in [the same month and year] and there's like, a ton of women in it. It was kind of reassuring, I didn't post a lot in the group and I still don't today, that everyone is kind of going through the same things and even now, if I don't know about things that are going on with the kids, knowing that you're not alone in this and maybe like, 'oh hey, people had it worse than I do.' So it's nice to know that somebody else has it too..." 
While social media did prove to be helpful for many, mass media appeared to have a negative effect on the way participants felt about their maternal bodies. Participant 3 noted that avoiding print media and augmented photographs of celebrities was something she found to be helpful:

"I really don't care about magazines. I think if I looked at that stuff, I would feel sad all of the time. And I don't. I choose not to look at stuff that's not that real..."

Normalization of their bodies was how most participants overcame body dissatisfaction and learned to cope with their new postbirth figures. Referring to the social and cognitive processes through which the motherly body comes to be seen as 'normal' or 'natural' in everyday life, normalization, in this context, served the purpose of preventing, minimizing, or reversing the effects of being viewed as different from society as a result of the changes endured during pregnancy and childbirth (May et al., 2009; Wolfensberger \& Tullman, 1982). The confirmation of her role as a mother and body's biologically reproductive purpose helped 5 participants cope with the changes they experienced. Participant 6 pointed to her biological role of reproduction as a means of normalizing her body changes, stating that:

"And the attitude that this is what my body was meant to do, it's not meant to be super model skinny. It was meant to bear children and that's what I wanted to do with it and that's what I did with it so..."

One participant even remarked that the changes of her body were "cool" and that having her child increased her confidence:

“... after having [my child], was I actually felt more confident. Like, I just had a baby. It's cool and okay if I'm not a perfect weight and that was kind of amazing. And it'll take a little time to get to a weight that I'm comfortable with and my body is working in a different way now."

Even despite complications with two pregnancies and births, as well as lasting consequences from child bearing, one participant described the joy that her children bring her as the best cure with the postpartum "baby blues." The love and compliments she receives from her 
children help her feel as though she has fulfilled her role as a mother despite the stretch marks and extra skin she has as visual reminders of her pregnancy. Her children tell her she is beautiful and that she is a good mom, and that affirmation is enough to overcome any dissatisfaction with her postbirth body.

Another participant, however, had a very different experience and the anxiety she experienced with pregnancy and after giving birth made motherhood difficult to cope with. She struggled with self-identification in the role of a mother as well as weight gain. Anxiety medication contributed to weight gain and made weight loss exponentially more challenging after giving birth.

One of the biggest challenges with pregnancy was a lack of honesty about the changes that one will experience during gestation and as a new mother. 3 participants believed they would have been better prepared for the changes their bodies underwent if other moms, books, and doctors had been more honest with them about the process. Not only did participants desire honesty about bodily changes, but also about what being a new mom is like and the amount of help they would actually need.

“Just more honest. Like, I wish the books would've, I wish it would've just not just talked about the process of labor. That's just a snapshot of time, you have the baby forever. You can get through the labor, I mean anybody can get through that, it might be painful, but it'll be over and then you have a baby, then what?"

\section{Discussion}

As seen in the interviews, discussing changes and experiences with other moms greatly impacted the process of accepting body changes after having a baby. Participants mentioned Facebook, forums, Google searches and Internet-based platforms as great resources for learning about the body changes they were enduring, and helping them overcome any dissatisfaction with 
their bodies. These findings were consistent with research done exploring the impact of Internet blogging on mothers. Self-proclaimed "Mommy bloggers" are able to share their personal stories with one another online, and provide reassurance to other mothers that their bodily changes and struggles are both normal and relatable (Husbands, 2008; Lopez, 2009).

While one participant did retrospectively cite photographing her pregnancy as a great way to embrace the changes, the majority of studies have revealed that in Western culture, there are primarily two kinds of photographs of pregnant women: medical pictures with obstetric characteristics and sexualized pregnancy images (Nash, 2014b). When sexy pictures of pregnant celebrities are released, they can elicit feelings of shame and guilt in other women, and often results in failing as a mother before even giving birth (Husbands, 2008). The specific and unrealistic idea frequently presented by the mass media of "yummy mummies"-women with unusually slender bodies, glowing skin, and bellies free of stretch marks during pregnancy—is far from the experience of most women during pregnancy (Husbands, 2008; Nash, 2012). By taking photographs of her own pregnancy to compare with one another, Participant 6 was able to compare the pictures amongst one another to see the changes instead of comparing herself to famous pregnant celebrities.

With this study, it is important to note that there are limiting factors to the generalizability of the results. One researcher, who also did all subsequent coding and analysis, created the thematic coding system used in this study. While this allowed for careful subjective analysis of the data, there is low inter-coder reliability of the data, and could be subject to the researcher's own experience and bias. Additionally, the group of participants in this study was too small to allow for results to be generalized to a greater population. While some moms may share similar thoughts and feelings to those interviewed in this study, it is extremely unlikely that 
the themes established in this research encompass the journey of all women who have given birth.

There are some limiting factors associated with the participant sample as well. As demographic was not collected from participants, there is no record of the age, race, or geographic location of the women who were interviewed. While the findings of this study are similar to those in studies of Australian women's post-birth body image, there is no verification that geographic location has an impact on body image, however the similarities in results do support the claim that women living in Western cultures have similar experiences of body image in postbirth (see Clark, Skouteris, Wertheim, Paxton, \& Milgrom, 2009a; Nash 2012; Nash, 2014a; Nash, 2014b). The lack of demographic information in combination with nature of a convenience sample may provide some limit to the number of women these results can be applied to.

It is also important to note that the name of this study has evolved as research was completed. The original title of this research study included the words dissatisfaction and body struggles, which were later deemed to be inaccurate descriptions of the research conducted. The former title was on the consent form participants received (see Appendix B), which may have had some influence on the information participants gave during the interview.

\section{Conclusion}

This research serves the purpose of bridging a gap in literature where the prebirth period meets the postbirth period. By exploring a greater period of time after birth than other studies, this research revealed more information about the long-term process of body change and reacceptance than studies that limited the postpartum timeframe to 3 to 12 months. Results were consistent with those of previous studies that examined experiences of body change with 
pregnancy, however this study focused primarily on learning about coping strategies women use to help them deal with the physical changes that accompany motherhood.

In general, normalization and support from loved ones and peers had the greatest impact on experiences with postbirth body changes. These coping strategies are not mutually exclusive and have many confounding variables however preliminary evidence in this field suggests that increasing the amount of peer support, both in-person and online, may have a positive impact on postbirth body changes. Far more research needs to be done to support this conjecture, including a greater number of interviews with a more diverse sample. In addition, by increasing the sample size, the risk of confidentially breach is minimized and it may be appropriate to collect demographic information, which could potentially increase the generalizability of results.

\section{Acknowledgements}

I would like to thank those who have supported me throughout this research process, starting with my advisor, Dr. Christopher Allen, who deserves the greatest thanks of all. His patience, guidance, and direction helped bring this research to fruition. I would also like to extend my gratitude to my family and friends whose continuous support has allowed me to overcome unforeseen obstacles. Finally, I want to thank my professors and mentors who have helped shape my knowledge and interest in academia. 


\section{References}

Cash, T., Pruzinsky, T. (1990). Body images: Development, Deviance, and Change. New York: Guilford.

Clark, A., Skouteris, H., Wertheim, E. H., Paxton, S. J., \& Milgrom, J. (2009a). My baby body: A qualitative insight into women's body- related experiences and mood during pregnancy and the postpartum. Journal of Reproductive and Infant Psychology, 27(4), $330-345$.

Clark, A., Skouteris, H., Wertheim, E. H., Paxton, S. J., \& Milgrom, J. (2009b). The relationship between depression and body dissatisfaction across pregnancy and the postpartum a prospective study. Journal of Health Psychology, 14(1), 27-35.

DePietro, J., Millet, S., Costigan, K., Gurewitsch, E., \& Caulfield, L. (2003.) Psychosocial influences on weight gain attitudes and behaviors during pregnancy. Journal of the American Dietetic Association, 103, 1314-1319.

Gow, R. W., Lydecker, J. A., Lamanna, J. D., \& Mazzeo, S. E. (2012). Representations of celebrities' weight and shape during pregnancy and postpartum: A content analysis of three entertainment magazine websites. Body Image, 9(1), 172-175.

Grbich, C. (2007). Qualitative data analysis. London: Sage.

Hoffnung, M. (1989). Motherhood: Contemporary conflict for women. Women: A Feminist Perspective, (157-170). Palo Alto, CA: Mayfield Publishing Company.

Hsieh, H., \& Shannon, S. (2005). Three approaches to qualitative content analysis. Qualitative Health Research, 15, 1277-1286.

Hueston, W. J., \& Kasik-Miller, S. (1998). Changes in functional health status during normal pregnancy. Journal of Family Practice, 47(3), 209-213.

Husbands, L. (2008). Blogging the maternal: Self-representations of the pregnant and postpartum body. Atlantis: Critical Studies in Gender, Culture \& Social Justice, 32(2), 68-79.

Jenkins, W. \& Tiggemann, M. (1997). Psychological effects of weight retained after pregnancy. Women and Health, 25, 89-98.

Jordan, K., Capdevila, R., \& Johnson, S. (2005). Baby or beauty: a Q study into post pregnancy body image. Journal of Reproductive and Infant Psychology, 23(1), 19-31.

Lopez, L. K. (2009). The radical act of 'mommy blogging': Redefining motherhood through the blogosphere. New Media \& Society, 11(5), 729-747. 
May, C., Mair, F., Finch, T., MacFarlane, A., Dowrick, C., Treweek, S... Montori, W. (2009). Development of a theory of implementation and integration: Normalization process theory. Implementation Science, 4(1), 29.

Nash, M. (2014a). Breasted experiences in pregnancy: An examination through photographs. Visual Studies, 29(1), 40-53.

Nash, M. (2014b). Picturing mothers: A photovoice study of body image in pregnancy. Health Sociology Review, 23(3), 242-253.

Nash, M. (2012). Weighty matters: Negotiation "fatness" and "in-betweenness" in early pregnancy. Feminism \& Psychology. 22(3), 307-323.

Nash, M. (2011). "You don't train for a marathon sitting on the couch": Performances of pregnancy 'fitness' and 'good' motherhood in Melbourne, Australia. Women's Studies International Forum, 34(1) 50-65.

Neuendorf, K. (2002). The content analysis guidebook. London: Sage.

Patel, P., Lee, J., Wheatcroft, R., Barnes, J., \& Stein, A. (2005). Concerns about body shape and weight in the postpartum period and their relation to women's self- identification. Journal of Reproductive and Infant Psychology, 23(4), 347-364.

Rallis, S., Skouteris, H., Wertheim, E. H., \& Paxton, S. J. (2007). Predictors of body image during the first year postpartum: A prospective study. Women \& Health, 45(1), 87104.

Thompson, J. K., Heinberg, L. J., Altabe, M., \& Tantleff-Dunn, S. (1999). Exacting beauty: Theory, assessment, and treatment of body image disturbance. American Psychological Association.

Tiggemann, M. (2004). Body image across the adult life span: Stability and change. Body Image, $1(1), 29-41$.

Walker, L. (1998). Weight-related distress in the early months after childbirth. Western Journal of Nursing Research, 20, 30-44.

Wolfensberger, W., \& Tullman, S. (1982). A brief outline of the principle of normalization. Rehabilitation Psychology, 27(3), 131-145. 


\section{Appendix A: Recruitment Transcript}

Good morning/afternoon/evening. My name is Samantha Lewis and I am an undergraduate student at the University Honors College at Portland State University in Portland, Oregon. I am working on my senior thesis under the supervision of Dr. Christopher Allen of the psychology department. Are you interested in learning more about this research?

If the answer is no, thank the individual for her time and end conversation. If the answer is yes, continue on.

The research I am conducting is centered on exploring some of the methods women use to overcome postbirth body dissatisfaction. Have you given birth within the past ten years?

If the answer is no, thank the individual for her time and end conversation. If the answer is yes, continue on.

Do you feel that your body has changed since you gave birth? Additionally, do you feel like you have come to terms with those changes in your body?

If the answer is no, thank the individual for her time and end conversation. If the answer is yes, continue on.

Because you have given birth within the past ten years and feel like you have come to terms with postbirth body changes, it sounds like you are a great fit for my research. Are you interested in learning about becoming a participant?

If the answer is no, thank the individual for her time and end conversation. If the answer is yes, continue on.

If you agree to become a participant, you will be asked to participate in a short, 30-minute interview conducted by me. Together, we will select a day and time within the next three weeks that works best for your schedule. The interview will take place in Dr. Allen's private office located at the Process Work Institute in Northwest Portland. There will be no compensation, monetary or otherwise, offered in exchange for your participation, however I am happy to share a copy of the final study with you when the research is complete. Would you like to be a research participant?

If the answer is no, thank the individual for her time and end conversation. If the answer is yes, provide them with a copy of the informed consent form and select a day and time to conduct the interview. 
Appendix B: Informed Consent

The Portland State University

Consent to Participate in Research

Body Dissatisfaction After Having Children: A Look into Postbirth Body Struggles Version 3: 07/21/17

You are being asked to participate in a research study that is being done by University Honors College student Samantha Lewis under the supervision of Dr. Christopher Allen at Portland State University in Portland, Oregon. This research is phenomenological and centered around exploring some of the methods women use to overcome postbirth body dissatisfaction.

You are being asked to participate in this study because you meet certain criteria set forth by the research team for this study. These criteria limit the research population to women who have given birth within the past ten years, and feel that they have come to terms with the changes their body has undergone since giving birth.

This form will explain the research study, as well as the possible risks and benefits to you. We encourage you to talk with your family and friends before you decide to take part in this research study. If you have any questions, please ask one of the study investigators.

If you agree to participate, the following things will happen: you will be asked to participate in a short, 30-minute interview conducted by Samantha Lewis. This interview will be recorded for later transcription. All recordings will be erased after transcription.

Participation in this study will take a total of approximately 30 minutes.

There are risks of stress and emotional distress associated with participating in a research study. For more information about risks and discomforts, ask the investigator.

We will take measures to protect the security of all your personal information, but we cannot guarantee confidentiality of all study data. To protect you identity and privacy, no personally identifying information will be used in the final research report/

Information contained in your study records will be used by the research team. The Portland State University Institutional Review Board (IRB) that oversees human subject research and/or other entities may be permitted to access your records, and there may be times when we are required by law to share your information. It is the investigator's legal obligation to report child abuse, child neglect, elder abuse, harm to self or others or any 


\section{life-threatening situation to the appropriate authorities, and; therefore, your confidentiality will not be maintained.}

Your name will not be used in any published reports about this study.

No monetary compensation will be offered for your participation in this study, however, if you'd like to receive a copy of the final study, please notify the research team.

Your participation in this study is completely voluntary. You have the right to choose not to participate or to withdraw your participation at any point in this study without penalty or loss of benefits to which you are otherwise entitled.

If you have any questions, concerns or complaints at any time about the research study, Dr. Chris Allen will be glad to attend to them at [email omitted]. If you need to contact someone after business hours or on weekends, please call [phone number omitted].

If you have questions regarding your rights as a research participant, you may call the PSU Office for Research Integrity at (503) 725-2227 or 1(877) 480-4400. The ORI is the office that supports the PSU Institutional Review Board (IRB). The IRB is a group of people from PSU and the community who provide independent oversight of safety and ethical issues related to research involving human participants. For more information, you may also access the IRB website at https://sites.google.com/a/pdx.edu/research/integrity. 


\section{PARTICIPANT CONSENT}

You are making a decision whether to participate in this study. Your signature below indicates that you have read the information provided (or the information was read to you). By signing this consent form, you are not waiving any of your legal rights as a research participant.

You have had an opportunity to ask questions and all questions have been answered to your satisfaction. By signing this consent form, you agree to participate in this study. A copy of this consent form will be provided to you.

Name of Adult Subject (print)

Signature of Adult Subject

Date

\section{INVESTIGATOR SIGNATURE}

This research study has been explained to the participant and all of his/her questions have been answered. The participant understands the information described in this consent form and freely consents to participate.

Name of Research Team Member (print) Signature of Research Team Member Date

\section{$\underline{\text { RESULTS }}$}

If you would like to receive the results of this study at the end of the process, please include your email address here:

Email 
Appendix C: Interview Guide

Interview Guide

Participant \#:

Tell me about the changes you experienced with your body during and after pregnancy.

How did you cope with those changes?

Was there something you did that you feel was helpful in adjusting to the changes in your body?

Other info given by participant: 